\title{
Review \\ Activator protein 1 (Fos/Jun) functions in inflammatory bone and skin disease
}

\author{
Rainer Zenz ${ }^{1,2}$, Robert Eferl ${ }^{1,2}$, Clemens Scheinecker ${ }^{3}$, Kurt Redlich ${ }^{3}$, Josef Smolen ${ }^{3}$, \\ Helia B Schonthaler ${ }^{4}$, Lukas Kenner ${ }^{1,2,5}$, Erwin Tschachler ${ }^{6}$ and Erwin F Wagner ${ }^{4 \dagger}$ \\ ${ }^{1}$ Ludwig Boltzmann Institute for Cancer Research, Währinger Strasse 13a, A-1090 Vienna, Austria \\ ${ }^{2}$ Center for Biomolecular Medicine and Pharmacy, Medical University of Vienna, Währinger Strasse 13a, A-1090 Vienna, Austria \\ ${ }^{3}$ Division of Rheumatology, Medical University of Vienna, Währinger Gürtel 18-20, A-1090 Vienna, Austria \\ ${ }^{4}$ Research Institute of Molecular Pathology, Dr. Bohr-Gasse 7, A-1030 Vienna, Austria \\ ${ }^{5}$ Clinical Institute of Pathology, Medical University of Vienna, Währinger Gürtel 18-20, A-1090 Vienna, Austria \\ ${ }^{6}$ Department of Dermatology, Medical University of Vienna, Währinger Gürtel 18-20, A-1090 Vienna, Austria \\ †Present address: Cancer Cell Biology Program, Spanish National Cancer Center (CNIO), Melchor Fernandez Almagro 3, E-28029 Madrid, Spain
}

Corresponding author: Erwin F Wagner, wagner@cnio.es

Published: 18 January 2008

This article is online at http://arthritis-research.com/content/10/1/201

(c) 2008 BioMed Central Ltd

Arthritis Research \& Therapy 2008, 10:201 (doi:10.1186/ar2338)

activating transcription factor and cAMP response elementbinding protein families also dimerize with the core members of the AP-1 family to regulate a broad variety of genes [2] by binding to their promoter and enhancer regions (Figure 1).

Although members of the Jun and Fos families share a high degree of structural homology, the individual AP-1 dimers exert significant differences in their DNA binding affinity and their capability to activate or suppress gene expression [3]. AP-1 converts extracellular signals of evolutionary conserved signaling pathways like mitogen-activated protein kinase, transforming growth factor beta and Wnt into changes in the expression of specific target genes that harbor AP-1 binding sites. Growth factors, neurotransmitters, polypeptide hormones, bacterial and viral infections as well as a variety of physical and chemical stresses employ AP-1 to translate external stimuli both into short-term and long-term changes of gene expression. These stimuli activate mitogen-activated protein kinase cascades that enhance AP-1 activity; for example, through phosphorylation of distinct substrates [4].

\section{Activator protein 1 functions in mice}

Many important insights regarding the specific functions of AP-1 proteins in development and disease have been obtained from genetically modified mice and the cells derived thereof (Table 1) [1,2]. In the following sections we shall present an overview of the different phenotypes obtained from gain-of-function and loss-of-function experiments, and we shall emphasize the lessons learned from these studies. 
Figure 1

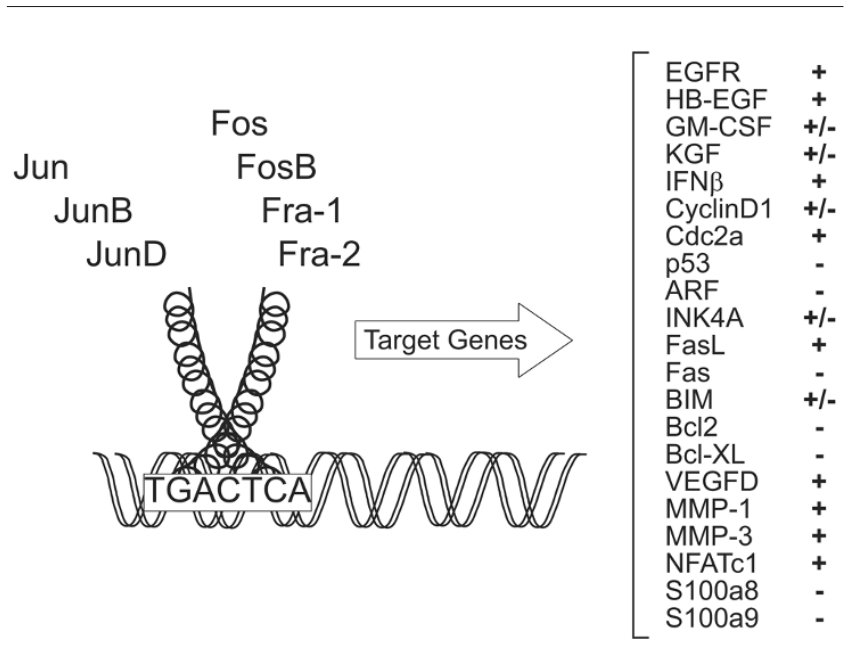

The activator protein 1 transcription factor. The dimeric activator protein 1 (AP-1) transcription factor is composed of Jun and Fos proteins. Jun proteins form homodimers or heterodimers with Fos proteins through their leucine-zipper domains. The different dimer combinations recognize different sequence elements in the promoters and enhancers of target genes. Only the classic TPA-responsive element with the consensus sequence TGACTCA is shown. The AP-1 dimers recognize the specific response elements via the basic domain that is adjacent to the leucine-zipper domain and represent an $\alpha$-helical structure. Among the target genes of AP-1 are important regulators of cell proliferation, differentiation and apoptosis. Some AP-1 targets are implicated in pathogenic processes such as $\mathrm{S100a8}$ and S100a9. Positively regulated $(+)$, negatively regulated $(-)$, or positively and negatively regulated (+/-) depending on the AP-1 dimer composition.

\section{Multiple roles of Jun proteins}

Transgenic expression of Jun, JunD or JunB in transgenic mice did not result in an overt phenotype, although targeted overexpression of JunB in $\mathrm{T}$ lymphocytes interfered with the differentiation of $\mathrm{T}$ helper cells ([1] and references cited therein), implying a role of JunB in $\mathrm{T}$ cell development. Ectopic expression of JunD under the control of the ubiquitin $\mathrm{C}$ promoter caused a reduction in the number of peripheral T cells and B cells, further suggesting a role of JunD in the regulation of the immune system ([1] and references cited therein). Jun was recently identified as a regulator of $\alpha \beta / \gamma \delta$ T-cell development by repressing IL7R $\alpha$ expression, which is essential for the $\gamma \delta$ lineage decision [5].

Jun and JunB are essential proteins for embryonic development, whereas JunD is required postnatally. Fetuses lacking Jun die between embryonic day 12.5 and embryonic day 14.5 of development with defects in liver development and heart morphogenesis [1]. Embryos lacking JunB show impaired vasculogenesis and angiogenesis in the extraembryonal tissue, leading to embryonic lethality around embryonic day 9.5 [6]. In contrast, mice lacking JunD are viable but exhibit reduced postnatal growth and multiple age-dependent defects in reproduction, hormone imbalance and impaired spermatogenesis [7].
A role for Jun/AP-1 in the control of cell proliferation has been proposed based on observations that AP-1 activity is induced upon mitogenic stimulation. Jun was shown to be primarily a positive regulator of cell proliferation. Jun-deficient fibroblasts have a marked proliferation defect in vitro, and proliferation of Jun-deficient hepatocytes was severely impaired during liver regeneration in vivo. Using conditional knockout techniques, we have recently shown that Jun/AP-1 regulates liver regeneration after partial hepatectomy through a novel molecular pathway that involves p53, p21 and the stress kinase p38 $\alpha$ [8]. Jun proteins need to be activated by Jun-amino-terminal kinases (JNKs) to fully promote cell-cycle progression. Once activated, Jun/AP-1 complexes induce the transcription of positive regulators of cell-cycle progression, such as cyclin $D_{1}$, or repress negative regulators, such as the tumor suppressor p53 and the cyclin-dependent kinase inhibitor p16 ${ }^{\text {INK4A }}$.

On the other hand, JunB and JunD are often considered negative regulators of cell proliferation. Fibroblasts overexpressing JunB showed reduced proliferation, whereas JunD-deficient immortalized fibroblasts exhibited increased proliferation [9]. Primary JunD-deficient fibroblasts also showed reduced proliferation, however, indicating that JunD can both positively and negatively regulate cell-cycle progression depending on the cellular context [10].

\section{Fos proteins in bone development and tumor formation} The expression pattern of Fos protein during embryonic mouse development indicated a possible role for the protein in endochondral ossification. Transgenic expression of Fos in many different cell types specifically affected the skeleton. In addition, chimeric mice obtained from Fos-overexpressing embryonic stem cells developed chondrogenic tumors, and ectopic expression of Fos from a ubiquitous promoter in transgenic mice resulted in the transformation of osteoblasts, leading to osteosarcomas [2]. Mice lacking Fos are viable and fertile but lack osteoclasts, resulting in an osteopetrotic phenotype ([2] and references cited therein).

Transgenic mice overexpressing $\Delta \mathrm{FosB}$, an isoform of FosB in osteoblasts, developed osteosclerosis with increased bone formation of the entire skeleton [11]. This phenotype is cell autonomous and is probably caused by enhanced differentiation and activity of osteoblasts. A similar osteosclerotic phenotype was observed in transgenic mice expressing Fra-1 in osteoblasts [12]. Ablation of Fra-1 during development resulted in lethality around embryonic day 10 due to placental defects, thereby preventing the analysis Fra-1 function in later development [2]. Applying conditional knockout techniques, we were recently able to demonstrate that mice lacking Fra-1 are viable and fertile but developed osteopenia, a low bone mass disease. Conditional Fra-1 knockout mice appeared to have normal numbers of osteoblasts and osteoclasts, but expressed reduced amounts of bone matrix components such as osteocalcin, collagen 1a2 and matrix Gla protein that are produced by osteoblasts and chondrocytes [13]. We 
Table 1

\begin{tabular}{|c|c|c|}
\hline Activator protein 1 protein & Phenotype & Affected organs/cells \\
\hline \multicolumn{3}{|l|}{ Transgenic } \\
\hline $\mathrm{H} 2 \mathrm{~Kb}$-Jun & None & None \\
\hline Ubiquitin C-JunBa & Increased bone mass & Not defined \\
\hline CD4-JunB & Enhanced T helper cell 2 maturation & Thymus, CD4 thymocytes \\
\hline Ubiquitin C-JunD & Peripheral $T$ cells and $B$ cells reduced & Lymphocytes \\
\hline $\mathrm{H} 2 \mathrm{~Kb}-\mathrm{Fos}$ & Osteosarcoma & Bone, osteoblasts \\
\hline $\mathrm{H} 2 \mathrm{~Kb}-$ Fos/Rsk-2-/y & Reduced osteosarcoma & Bone, osteoblasts \\
\hline H2Kb-FosB & None & Bone \\
\hline TCR $\beta-\Delta$ FosB & Impaired T cell differentiation & Thymus, immature thymocytes \\
\hline NSE- $\Delta$ FosB & Osteosclerosis & Bone, osteoblasts \\
\hline $\mathrm{H} 2 \mathrm{~Kb}-\mathrm{Fra}-1$ & Osteosclerosis & Bone, osteoblasts \\
\hline CMV-Fra-2 & Occular malformations & Anterior eye structure \\
\hline $\mathrm{H} 2 \mathrm{~Kb}-\mathrm{Fra} 2^{\mathrm{a}}$ & Increased bone mass, fibrosis & Bone, internal organs, skin \\
\hline \multicolumn{3}{|l|}{ Knockout } \\
\hline Jun & Embryonic lethal on embryonic day 12.5 & Liver, heart, neural crest \\
\hline JunB & Embryonic lethal on embryonic day 10 & Extraembryonic tissues \\
\hline JunD & Male sterility & Testis, spermatides \\
\hline c-Fos & Osteopetrosis & Bone, osteoclasts \\
\hline FosB & Nurturing defect & Brain, hypothalamus \\
\hline Fra-1 & Embryonic lethal on embryonic day 9.5 & Extraembyonic tissue \\
\hline Fra-2 & Lethal at birth & Bone, osteoclasts \\
\hline \multicolumn{3}{|l|}{ Conditional } \\
\hline Alfp-cre Jun & Liver regeneration defect & Liver, hepatocytes \\
\hline Col2a1-cre Jun & Scoliosis & Bone, notochordal cells \\
\hline Nestin-cre Jun & Axonal regeneration defect & Central nervous system, motoneurons \\
\hline MORE-cre JunB & Osteopenia & Bone, osteoclasts, osteoblasts \\
\hline K5-cre Jun & Eyes open at birth, reduced skin tumors & Keratinocytes \\
\hline Nestin-cre Fos & Learning defects & Brain, hippocampal neurons \\
\hline MORE-cre Fra-1 & Osteopenia & Bone, osteoblasts \\
\hline \multicolumn{3}{|l|}{ Inducible } \\
\hline K5-creERTJunB + Jun & Psoriasis-like disease & Skin, joints, keratinocytes \\
\hline
\end{tabular}

Knockout, conditional knockout and gain of function (transgenic) approaches applied to study the role of Jun and Fos proteins during development and in diseases. The gain-of-function approaches were performed with different promoters, either leading to ubiquitous expression (for example, $\mathrm{H} 2 \mathrm{~Kb}$, ubiquitin $\mathrm{C}$, or cytomegalovirus (CMV)) or to tissue-specific expression (for example, CD4, TCR $\beta$, or neuron-specific enolase (NSE)) of the transgenes. aUnpublished data from the Wagner Laboratory.

therefore speculate that Fra-1 functions in bone forming osteoblasts, mainly by affecting the activity of the cells through the regulation of matrix production and not by affecting the proliferation or differentiation of cells.

Mice overexpressing Fra-2 under the control of a cytomegalovirus promoter were reported to display ocular malformations due to disrupted development of anterior eye structures [14]. When Fra-2 was broadly expressed from the $\mathrm{H} 2$ promoter in many organs, however, the mice developed a severe fibrotic disease mostly in the lung, as well as occasional fibrosarcomas, alongside an increased bone mass (A Bozec, R Eferl, P Hasselblatt, unpublished data). In contrast, the absence of Fra-2 in embryos and newborn mice affected hypertrophic chondrocyte differentiation and matrix production [15], and mutant pups died shortly after birth [16]. Moreover, Fra-2 knockout newborns exhibited cell autonomous defects in osteoclasts and osteoblasts that were dependent on signaling from the LIF/LIF-receptor system (A Bozec, L Bakiri, unpublished data). Chondrocyte-specific 
inactivation of Fra-2 led to cell autonomous defects in cartilage, since mutant mice were growth retarded and developed a kyphosis-like phenotype [15]. Interestingly, mice lacking JunB are also osteopenic due to cell-autonomous osteoblast and osteoclast defects [17].

Taken together, Fos/AP-1 proteins are important regulators of bone formation, and therapeutic interventions acting on AP-1 signaling might provide a powerful approach for the treatment of low bone mass diseases.

\section{Activator protein 1 in inflammation}

Chronic inflammatory diseases, such as inflammatory bowel disease, chronic obstructive pulmonary disease, rheumatoid arthritis (RA), psoriasis and psoriatic arthritis, are affecting a large segment of the population. In addition, cancer and even metabolic diseases, such as type 2 diabetes or atherosclerosis, are believed to have an inflammatory component [18]. It is thought that in several of these diseases chemotactic/chemoattractant proteins and cytokines are released at the side of injury or infection, which then attracts innate and adaptive immune cells. The cytokine milieu together with the immune cells triggers a cascade of events, called the inflammatory process. Interestingly, many cytokine genes are regulated cooperatively by a transcription factor complex consisting of AP-1 and nuclear factor of activated T cells (NFAT). NFAT-dependent gene regulation has been demonstrated for IL-2, IL-3, granulocyte-macrophage colonystimulating factor, IL-4, IL-5, IL-13, IFN $\gamma$, TNF $\alpha$, CD40L, FasL, CD5, Igא, CD25 and the chemokines IL-8 and MIP1 $\alpha$. Importantly, for the majority of these genes, the induction with AP-1 appears essential.

The innate immune system employs cellular components such as macrophages or dendritic cells and humoral components of the complement system to respond to infectious agents. The activation of Toll-like receptors is an important starting point for the activation of innate immunity. Once activated, Toll-like receptors lead among other events to the differentiation of macrophages and to the production of several cytokines such as TNF $\alpha, \mathrm{IL}-1, \mathrm{IL}-6$ or IL-12. The signaling of Toll-like receptors leading to cytokine production is integrated by adapter molecules such as MyD88 and TRAF6 that eventually activate NF-KB and AP-1 [19].

Allergic asthma, RA and psoriasis are thought to be inflammatory diseases mediated by activated T cells. AP-1 has been shown to be involved in the differentiation of naïve T cells into T helper 1 cells and T helper 2 cells, which is a hallmark of the $T$ cell-dependent immune response. JunB positively regulates IL-4 expression and accumulates in Thelper 2 cells during differentiation [20]. In agreement, loss of JunB in polarized T helper 2 cells in vitro is followed by deregulated expression of T-helper-2-specific cytokines and by expression of IFN $\gamma$ and T-bet, which are known as key regulators of $\mathrm{T}$ helper 1 cells [21]. The molecular mecha- nisms by which Jun and JunB regulate $T$ helper 2 cytokine expression has been identified recently. The turnover of Jun and JunB is regulated by ubiquitin-dependent proteolysis after targeting for degradation by the E3-ligase Itch in a JNKdependent pathway [22]. In contrast, ectopic overexpression of JunD suppresses $T$ cell proliferation and activation due to reduced expression of IL-4, CD25 and CD69 [23]. Together, these data implicate Jun proteins as important players in T cell-mediated diseases that are characterized by an imbalanced ratio of $\mathrm{T}$ helper 1 effector cells and $\mathrm{T}$ helper 2 effector cells.

Glucocorticoids are very effective in controlling inflammation and are used for the treatment of autoimmune diseases such as RA. Expression of several cytokines such as IL-1, IL-2 or IFN $\gamma$ is activated by AP-1 and other transcription factors, but is repressed by the glucocorticoid receptor (GR). Recent data suggest that the GR prevents the interaction between DNA-bound AP-1 complexes and transcriptional coactivators. Irrespective of the exact mechanism, the ability of the GR to repress the proinflammatory transcription factors AP-1 and NF- $\kappa B$ seems the most important function of the GR. This has been demonstrated with genetically modified GRdim/dim mice, whose GR is unable to bind to GR-responsive DNA elements but is still capable of transrepressing AP-1 and NF-KB [24].

\section{Functions of activator protein 1 in the pathogenesis of inflammatory bone diseases}

Bone is a highly dynamic organ that is continuously remodeled by osteoclasts and osteoblasts. Any disturbance in the balance between these cells causes a pathogenic change in bone mass. This could either be a loss of bone mass as observed in postmenopausal osteoporosis or a gain of bone mass as observed in osteopetrosis. Evidence from a variety of mouse models suggests that the AP- 1 transcription factor is directly or indirectly implicated in the development of several bone diseases [2]. AP-1 influences the pathogenic outcome of bone diseases not only via differentiation of bone cells but also via inflammatory processes. We shall focus on two types of inflammatory diseases, RA and psoriatic arthritis, and shall discuss the potential role of the AP-1 transcription factor.

\section{Rheumatoid arthritis and activator protein 1}

$\mathrm{RA}$ is considered an autoimmune disorder where the immune system preferentially attacks the joints. Extraarticular tissues such as skin, blood vessels, the heart, the lungs and muscles, however, can also be affected in a systemic manner. Besides aging, several risk factors have been identified, such as gender, environmental conditions and genetic predisposition. In addition, a strong genetic association between the major histocompatibility complex antigen DR4 and the prevalence for RA has been observed [25].

Histopathologically, RA is characterized by synovial inflammation, cartilage destruction and erosion of subchondral 
bone, eventually leading to a substantial loss of joint mobility. Activated $\mathrm{T}$ cells are considered the major inflammatory component that affects the severity of RA [26]; however, others see cells of the monocyte/macrophage lineage or synovial fibroblasts as the main culprits [27]. The cellular mechanism by which $T$ cells promote joint destruction in RA has been unravelled using different animal models. For example, collagen-induced arthritis has been widely used as an animal model for RA. The disease is induced by immunization of mice or rats with type II collagen and an adjuvant.

RA is also characterized by the overexpression of proinflammatory cytokines. In fact, a particularly important genetic model that was used to investigate the cellular interactions in RA is transgenic mice expressing human TNF $\alpha$ from a globin promoter (hTNFtg mice). The hTNFtg mice develop a RA-like disease that is characterized by inflammation of the joints, joint swelling and bone erosions (see later Figure 4e,f). Breeding of hTNFtg mice with knockout mice lacking the AP-1 component Fos, and therefore devoid of osteoclasts, demonstrated the essential requirement for osteoclasts in RA. hTNFtg Fos ${ }^{-/-}$mice are completely protected from hTNFtg-induced bone erosion, although the severity of synovial inflammation as well as paw swelling and the reduction of grip strength were not ameliorated. Similar studies where osteoprotegerin was used to inhibit osteoclast differentiation suggest that activated cells present in the rheumatoid synovial membrane, such as T cells or fibroblasts, promote Fos-dependent differentiation of macrophage precursors into osteoclasts, thereby promoting bone resorption [28].

One key signaling molecule that was initially identified on activated $T$ cells and as a regulator of $T$ cell function is the receptor activator of NF- $\mathrm{KB}$ ligand (RANKL) - also called TRANCE, ODF, OPGL or TNFSF11 [29]. Under pathogenic conditions such as RA, RANKL is also secreted by a variety of synovial cells including inflammatory $T$ cells, thereby promoting extensive osteoclastogenesis and bone resorption [30]. One potent negative regulator of RANKL is the decoy receptor osteoprotegerin, which competes with RANKL for binding to the receptor activator of NF- $\mathrm{KB}$ receptor on osteoclast precursors, thereby inhibiting RANKL-induced osteoclastogenesis [31]. In RA, however, the ratio between RANKL and osteoprotegerin is shifted in favor of RANKL, resulting in a net increase of osteoclastogenesis. Based on this knowledge, a human anti-RANKL antibody called Denosumab has been developed and is currently being tested for treatment of postmenopausal osteoporosis as well as of local bone erosions in RA [32].

The most important transcription factor complexes that are activated by RANKL/TRAF signals are NF- $\kappa B$ and Fos/AP-1 [2]. The inactivation of NF- $\kappa B$ or Fos causes severe osteopetrosis due to the lack of osteoclasts. Two key target genes of Fos in osteoclastogenesis have been identified recently.
The first gene, NFATc1, turned out to be a promoter of osteoclatogenesis, whereas the second gene, IFN $\beta$, is an antagonist. NFATc1 is not solely a downstream target of Fos but also cooperates with Fos and Jun proteins to induce osteoclast-specific genes such as tartrate-resistant acid phosphatase or cathepsin K. Most importantly, ectopic expression of NFATc1 can rescue the osteoclast differentiation defect of Fos-deficient monocyte precursors, suggesting it is the most critical target gene of Fos in osteoclastogenesis [33]. The other Fos target gene that is activated by RANKL is IFN $\beta$. Surprisingly, IFN $\beta$ has been shown to reduce the expression of Fos in osteoclast precursors. This has led to a model where IFN $\beta$ provides a negative feedback loop that prevents extensive osteoclastogenic activity of Fos [34]. The implication of NFATc1 and IFN $\beta$ in RA is very likely, since these proteins are key target genes of Fos. Further studies are required, however, before their potential use as therapeutic targets is taken into account.

AP-1 activity can also affect the severity of RA at a level different from osteoclastogenesis. In addition to ostecoclastmediated bone erosion, several molecules are secreted by synovial fibroblasts that contribute to matrix degradation. Of particular importance are matrix metalloproteinases (MMPs) that are regulated by $\mathrm{AP}-1$ and degrade collagen, fibronectin or other components of the extracellular matrix. The major MMPs that are implicated in RA are MMP-1, MMP-9, MMP-13 and MMP-14 (MT1-MMP) [35]. These MMPs are expressed by activated osteoclasts or by synovial fibroblasts, or by both. The significance of AP-1-mediated MMP regulation in RA, however, has not yet been demonstrated in suitable mouse models.

Signals that lead to activation of Jun have been implicated in RA. In particular, JNK is highly activated in synovial fibroblasts of RA. The use of the JNK inhibitor SP600125 blocked accumulation of phospho-Jun in synovial fibroblasts, reduced the expression of the Jun target gene collagenase-3 and ameliorated bone erosion after collagen-induced arthritis in rats [36]. JNK/Jun signaling should therefore also be considered a potential therapeutic target for RA.

In summary, AP-1 activity is induced in RA by inflammatory cytokines and has a complex impact on osteoclast differentiation and production of soluble mediators of bone erosion. It can be anticipated that several AP-1 components or signaling pathways leading to AP-1 activation may provide valuable drug targets for therapy of RA in the future. At present, however, therapies that target TNF- $\alpha, \mathrm{IL}-1, \mathrm{IL}-6, \mathrm{~B}$ cell and $T$ cell costimulation are the most effective biological treatments [37].

\section{Activator protein 1 and epidermal disease}

AP-1 has been proposed to play important functions in the epidermis of the skin, from differentiation to wound repair and carcinogenesis. Conditional, epidermis-specific knockout 

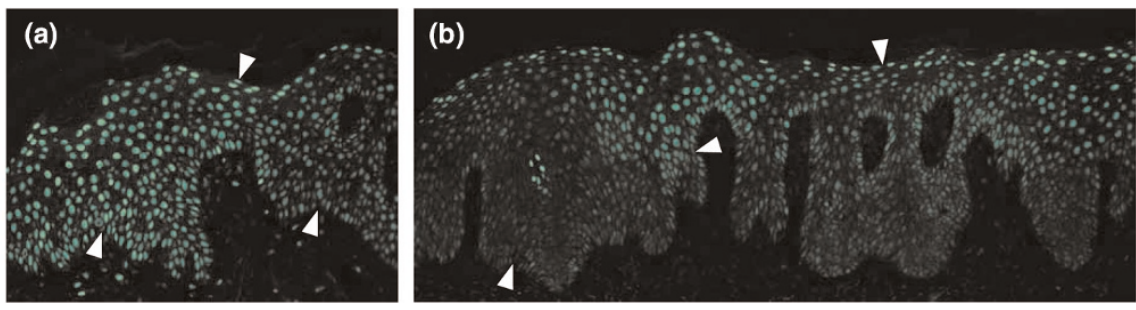

Heterogeneous JunB expression within lesional psoriatic skin. Immune reactivity of a monoclonal antibody against JunB within a psoriatic lesion. (a) Distinct anti JunB reactivity of a parakeratotic lesion. JunB expression is observed throughout all epidermal layers (left side, arrow), whereas it is reduced on the right side of the lesion (see arrow). (b) A different area of the same lesion. A virtual absence of nuclear reactivity is seen in basal keratinocytes, whereas strong nuclear activity is detected in the upper suprabasal epidermal layers ((a) and (b) arrows).

mice recently provided insights into the function of Jun/AP-1 in skin biology in vivo [1]. Jun is regarded a positive regulator of keratinocyte proliferation/differentiation through its direct transcriptional effect on epidermal growth factor receptor expression [38]. In contrast, JunB can antagonize the proliferation of keratinocytes and hematopoietic stem cells. Adult mice lacking JunB in the epidermis developed skin ulcerations in the facial area, hypergranulopoiesis and lost bone mass, most probably mediated by granulocyte colonystimulating factor release from the epidermis (A Meixner, R Zenz, HB Schonthaler, L Kenner, H Scheuch, JM Penninger, EF Wagner, manuscript under revision). Epidermis-specific deletion of JunB therefore appears to affect distant organs affecting myelopoiesis and bone homeostasis, supporting the notion of an endocrine-like function of the skin.

\section{Psoriasis and the activator-protein-1-dependent mouse model}

Psoriasis is a chronic inflammatory skin disease affecting $1-3 \%$ of the general population. At the histopathological level the disease is characterized by accelerated proliferation and altered differentiation of keratinocytes and extensive mixed leukocyte infiltrates consisting of $\mathrm{T}$ cells, monocytes and neutrophils [39]. In up to $40 \%$ of patients, the skin disease is associated with arthritis [40]. The beneficial therapeutic effects of immunosuppressive drugs such as cyclosporine as well as the new class of 'biological agents' have established a central role of immune cells in the pathogenesis of psoriasis [39]. It is still controversial, however, whether the involvement of immune cells is the cause of or the consequence of the psoriasis phenotype observed in keratinocytes [41]. Although at least six different psoriasis susceptibility loci (PSORS1-PSORS6) have been mapped in the human genome, the genetic basis of psoriasis remains largely unknown [42].

We recently described that expression of human JunB, which is localized in the PSORS6 locus (psoriasis susceptibility locus 6 ; 19p13), was reduced in lesional areas of severe psoriasis, suggesting a possible role of JunB in the develop- ment of the disease [43]. Moreover, reduced AP-1 binding activity was also reported in lesional skin from psoriatic patients [44]. In contrast, others reported a slight but insignificant increase of JunB mRNA and protein expression in psoriasis vulgaris lesions [45], and argue that induced JunB expression in keratinocytes may be part of an overall inflammatory response. We recently found that there is heterogeneity in the expression of JunB within lesional skin (Figure 2), but JunB expression also seems to be variable between individuals in nonlesional skin. It is presently unclear whether these differences in gene expression are caused by the heterogeneity and complexity of the disease. Additional experiments with human samples as well as human keratinocyte cultures are necessary to establish the role of JunB in skin inflammation and whether modulation of JunB expression is associated with the pathogenesis of the disease.

To downregulate Jun/AP-1 expression in the epidermis of adult mice, we generated epidermis-specific, inducible singleknockout and double-knockout mice for JunB and Jun (Figure 3a). Mice harboring conditional JunB and Jun alleles were crossed to $\mathrm{K} 5-\mathrm{Cre}-\mathrm{ER}^{\top}$ transgenic mice, in which tamoxifen efficiently induced Cre-mediated deletion of JunB and/or Jun in the basal layer of the epidermis. Adult singlemutant and double-mutant mice and their littermate controls were injected with tamoxifen and monitored for 14 days (Figure 3b). Inducible deletion of JunB or Jun in the epidermis revealed no signs of a skin phenotype up to 2 months after deletion. Interestingly, JunB/Jun double-mutant mice developed skin alterations mainly affecting hairless skin, which resemble lesions observed in patients with psoriasis. One hundred percent of the double-mutant mice showed a strong phenotype with inflamed scaly plaques affecting primarily the ears, paws and tail, and less frequently the hairy back skin after 3 weeks (Figure $3 c-h$ ). The affected skin of doublemutant mice showed the hallmarks of psoriasis, with a strongly thickened epidermis, hyperkeratosis (thickened keratinized upper layers) with nucleated keratinocytes in the cornified layer (parakeratosis) and increased subepidermal vascularization (Figure 3e,f). Intraepidermal T cells, epidermal 
microabscesses and the typical inflammatory cell infiltrate consisting of neutrophils were seen together with increased numbers of macrophages in the dermis. Arthropathic lesions seen in 5-40\% of psoriasis patients were observed in double-mutant mice with inflammatory infiltrates in the joint regions along with bone destruction and periostitis (see below) [43].

Since many of the histological and molecular hallmarks of psoriasis are reproduced in mice with epidermal deletion of JunB and Jun, we employed this mouse model to address the role of immunocytes during disease development. JunB and Jun were therefore deleted in mice deficient for Rag2 that lack functional $\mathrm{T}$ cells and B cells. Interestingly, the skin phenotype of Rag2-deficient JunB/Jun double-mutant mice was milder but still present when compared with JunB/Jun double-mutant mice, suggesting a minor role for $\mathrm{T}$ cells and $B$ cells in the etiology of the skin disease in this model. Arthritic-like lesions were almost absent in these mice, however, strongly implicating the involvement of $\mathrm{T}$ cells in the development of the phenotype [43]. It will be interesting to analyze in detail the immunocyte subsets to further explore the role of macrophages and dendritic cells. Both cell types might contribute to the production of TNF $\alpha$, which was still highly expressed in the epidermis even in the absence of functional T cells.

Recently developed biological agents are directed towards inhibiting TNF $\alpha$ signaling. We therefore genetically deleted JunB and Jun in TNFR1 knockout mice. Interestingly, this deletion did not prevent the development of the skin phenotype, although histological analyses showed a milder phenotype when compared with JunB/Jun double-mutant mice. The inflammation of the joint regions was again almost absent, demonstrating a functional contribution of TNF $\alpha$ signaling via TNFR1 to the etiology of the joint lesions.

Another key finding in double-mutant mice was the rapid upregulation of genes encoding the $\mathrm{Ca}^{2+-}$-binding proteins S100a8 and S100a9 in keratinocytes upon deletion of JunB and Jun, both in vivo and in vitro. The S100a8 and S100a9 genes map to the PSORS4 region and have been found strongly upregulated in affected areas of psoriatic skin. The S100a8/S100a9 complex functions as a chemotactic signal for $T$ cells and neutrophils. S100a9 knockout mice are viable and fertile but do not form S100a8/S100a9 complexes [46]. These mice are currently employed in our laboratory to test the functional contribution of S100a8/S100a9 in disease development. Preliminary results suggest that S100a8/S100a9 may indeed be an important signal early in the development of the phenotype, since the disease phenotype appears to be altered in mice lacking S100a9 (HB Schönthaler, EF Wagner, unpublished data).

The mouse model lacking JunB and Jun in the epidermis largely recapitulates the histological and molecular hallmarks
Figure 3

(a)
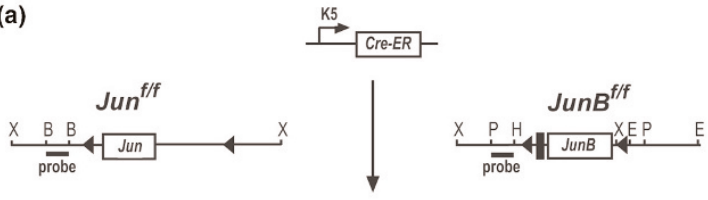

Cre-ER protein

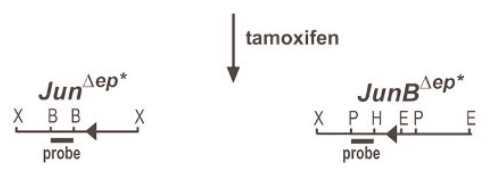

(b)
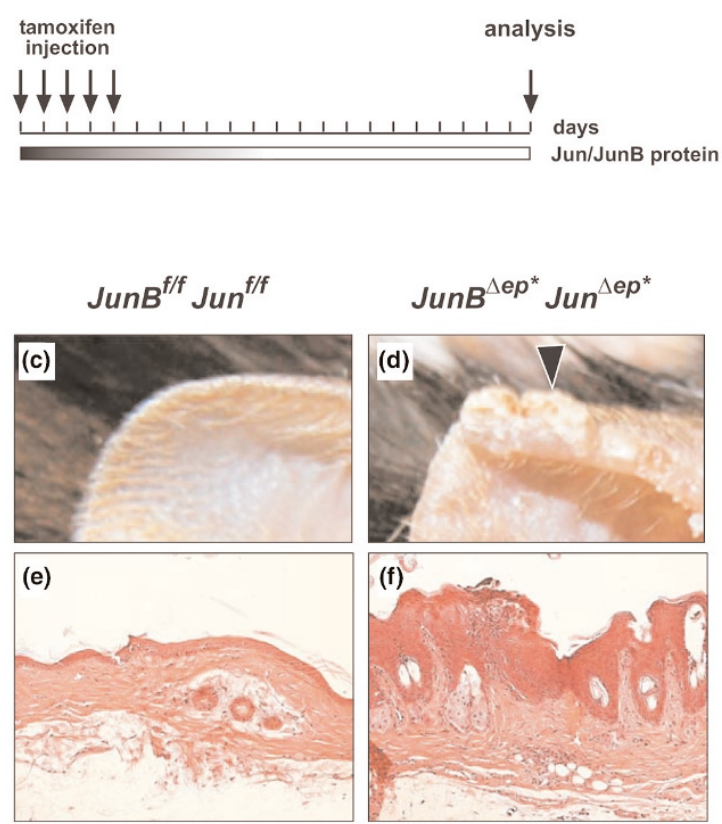

$\operatorname{JunB}^{\Delta e p^{*}} \operatorname{Jun}^{\Delta e p^{*}}$
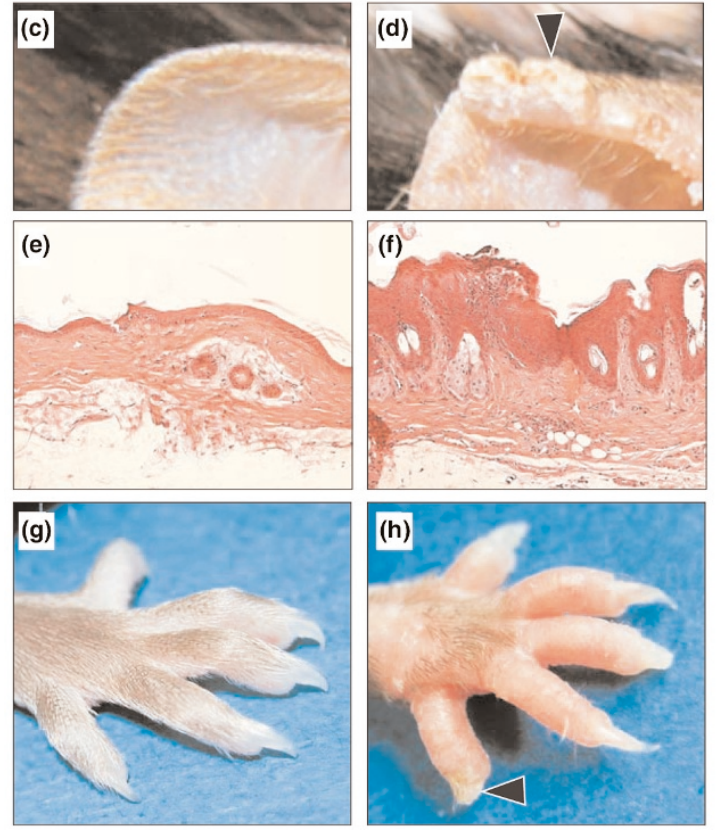

Inducible deletion of JunB and Jun and in the epidermis of adult mice. Mice carrying floxed alleles for the Jun and/or JunB locus were used to delete one or both genes in the epidermis by inducible Crerecombinase activity. (a) Schematic representation of the floxed Jun and JunB loci before and after tamoxifen-induced activation of the CreER-fusion protein, which is under the control of the keratin 5 promoter. (b) Eight-week-old mice were injected for five consecutive days with tamoxifen to activate Cre-mediated deletion of Jun and JunB. Two weeks after the last injection ((c), (e) and (g), control mice), the double-mutant mice $((\mathrm{d}),(\mathrm{f})$ and $(\mathrm{h}))$ showed a strong skin and arthritis phenotype reminiscent of psoriasis mainly affecting the (d) ears, ( $f$ ) tail and (h) paws. H\&E staining of (e) control mice and (f) mutant skin mice reflects the histological hallmarks of psoriasis with abnormally thickened epidermis, parakeratosis, hyperkeratosis and fingerlike epidermal projections into the dermis. 

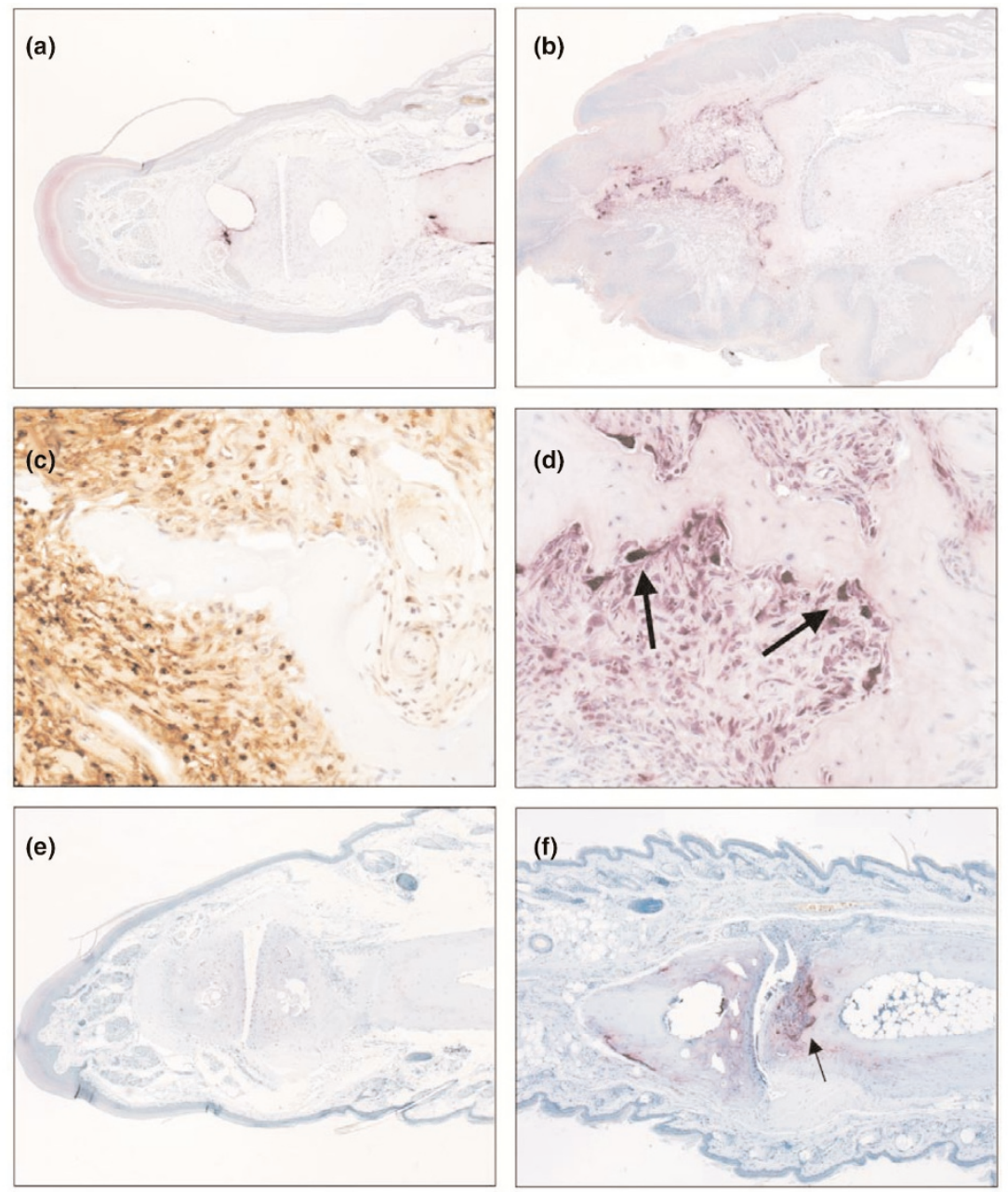

Distinct joint pathology in an inducible Jun mouse model of psoriasis. Microscopic images of a mouse toe from (a) a wildtype littermate control and (b)-(d) JunB/Jun double-mutant mice. Tartrate-resistant acid phosphatise-stained paraffin sections demonstrate (b) a proliferative periostitis affecting both the underlying bone as well as (c) the overlying nail base and dermis with numerous infiltrating neutrophilic granulocytes (antineutrophil NEU47 staining). In advanced stages, (b) an almost complete destruction of the distal phalanx and (d) bone erosions with osteoclasts invading the bone tissue (arrows) can be observed. In contrast, in transgenic mice expressing human TNF $\alpha$, no destruction of the distal phalanx and no erosive arthritis of the distal interaphalangeal joints are found: (e) wildtype control and (f) tartrate-resistant acid phosphatase staining. (f) Pannus formation and osteoclast-mediated subchondral bone destruction, similar to human rheumatoid arthritis, is consistently observed (arrow). Magnification: (a), (b), (e) and (f), $50 \mathrm{x}$; (c) and (d), $200 \mathrm{x}$.

seen in psoriasis. Previous attempts to reproduce the psoriatic phenotype by expression of inflammatory mediators or growth factors such as TNF $\alpha, \mathrm{IL}-1 \beta, \mathrm{IFN} \gamma$, keratinocyte growth factor, vascular endothelial growth factor, transforming growth factor beta 1, Stat3 and others (reviewed in [47]) yielded also phenotypes partially resembling psoriasis. Moreover, almost all of the mouse models discussed above showed no arthritic lesions [48].

\section{Psoriasis-like arthropathy in the inducible, epidermis-specific Jun mouse model}

The psoriasis-like disease in JunB/Jun double-mutant mice is characterized by periarticular inflammation with an asym- metric pattern of involvement. The first clinical signs of the disease are an elevation and thickening of the nails accompanied by sausage-like swelling of one or more toes, which are not always uniformly affected (Figure 3h). Different manifestations of the disease, such as synovitis, dactylitis and enthesitis - all of which occur rapidly - were recognized by microscopic analysis (L. Kenner, unpublished data). Moreover, the severe form of the disease involved individual toes with shortening and thickening of the distal phalanx covered by hyperkeratotic, edematous skin. Distal interphalangeal joints and cartilage were only mildly affected. The 'sausage' digit was characterized by extensive subcutaneous edema accompanied by a proliferation of small blood vessels and an 
acute inflammatory reaction involving numerous neutrophils. Inflammatory infiltrates were observed within the proliferating and thickened synovial lining layer with profound lymphocytic and granulocyte infiltration as well as the presence of small vessels (L. Kenner, unpublished data). Tenosynovitis with perimuscular and tendon sheath edema as well as cell infiltrations were also seen. The proliferative periostitis affected both the underlying bone and the overlying nail base in a continuous process. The overlying dermis appeared also with a mixed infiltrate, since the dermis was edematous and hyperplastic.

These changes described above are reminiscent in their severity to inflammatory skin infiltrates. In advanced stages, dactylitis led to an almost complete destruction of the distal phalanx (Figure 4b). Osteoclasts invading the bone were observed at the front of erosions and suggested a periosteum-derived, sometimes granulomatous, tissue (Figure 4d). It is worth pointing out that these manifestations are different from the joint pathology observed in the hTNFtg mouse model of RA [49].

As in human RA, no destruction of the distal phalanx can be seen in hTNFtg mice and the erosive arthritis typically spares the distal interaphalangeal joints (Figure 4f). Moreover, pannus formation and osteoclast-mediated subchondral bone destruction is prominent in hTNFtg mice (Figure 4f).

The histopathology of JunB/Jun double-mutant mice differs from human RA but is reminiscent of a rare form of psoriasis pustulosa called akrodermatitis continua suppurativa Halopeau [50]. In this disease lesions typically develop on the distal portion of the digits, involve the nail bed and spread proximally with time, finally leading to onychodystrophy [51]. The relationship between skin and nail involvement and joint manifestations is not resolved [51]. A detailed analysis at different time points during disease progression starting from toe involvement until joint disease could certainly help to clarify this question.

\section{Conclusions}

AP-1 is considered a transcription factor of general importance for many cellular processes in different organs. It was therefore somewhat surprising that gene knockout experiments demonstrated rather tissue-specific and cellspecific functions of individual AP-1 components, particularly in development. Some of these specific functions from conditional AP-1 knockout studies are implicated in diseases that are linked to inflammatory processes such as RA or psoriasis. Under these circumstances, AP-1 might be implicated as a downstream mediator of cytokine signaling. Alternatively, deregulated AP-1 activity might directly be causally involved in the initiation of disease development before inflammation takes place. The latter possibility is convincingly demonstrated in the psoriasis-like mouse model with deletions of JunB and Jun in epidermal cells. Such mouse models are essential to dissect the molecular pathways that lead to various organ-specific phenotypes that can be observed in more complex diseases. These models can also be employed for preclinical studies with known or novel therapeutic drugs, and they may reveal unexpected environmental factors that have not been considered in diseases such as psoriasis. For example, we have obtained preliminary data in the psoriasis mouse model suggesting that ciprofloxacin significantly delayed the onset of the skin disease and prevented the arthritic-like phenotype. This observation implies that resident bacteria might contribute to the manifestation of the joint disease.

It is plausible that different molecular pathomechanisms are responsible for the organ-specific manifestations of complex diseases. This would imply that therapeutic strategies have to be custom-tailored for each mechanism and used in a combinatorial manner to give attribute to all disease manifestations. Alternatively, identification of factors and pathways such as AP-1 that could be directly involved in diseases such as psoriasis may offer the possibility for a target-directed therapy.

\section{Competing interests}

The authors declare that they have no competing interests.

\section{Acknowledgements}

The authors are very grateful to members of the Wagner laboratory for critical reading of the manuscript and helpful comments, and they thank Hannes Tkadletz for help in preparing the illustrations. RZ, RE, and LK are funded by the Ludwig Boltzmann Society. RE is also funded by the SFB grant SFB-F28. The Research Institute of Molecular Pathology is funded by Boehringer Ingelheim, and the present work was supported by the Austrian Industrial Research Promotion Fund.

\section{References}

1. Zenz R, Wagner EF: Jun signalling in the epidermis: from developmental defects to psoriasis and skin tumors. Int $J$ Biochem Cell Biol 2006, 38:1043-1049.

2. Wagner EF, Eferl R: Fos/AP-1 proteins in bone and the immune system. Immunol Rev 2005, 208:126-140.

3. Shaulian E, Karin M: AP-1 as a regulator of cell life and death. Nat Cell Biol 2002, 4:E131-E136.

4. Chang L, Karin M: Mammalian MAP kinase signalling cascades. Nature 2001, 410:37-40.

5. Riera-Sans L, Behrens A: Regulation of alphabeta/gammadelta $\mathrm{T}$ cell development by the activator protein 1 transcription factor c-Jun. J Immunol 2007, 178:5690-5700.

6. Schorpp-Kistner M, Wang ZQ, Angel $P$, Wagner EF: JunB is essential for mammalian placentation. EMBO $J$ 1999, 18:934948.

7. Thepot D, Weitzman JB, Barra J, Segretain D, Stinnakre MG, Babinet $C$, Yaniv M: Targeted disruption of the murine junD gene results in multiple defects in male reproductive function. Development 2000, 127:143-153.

8. Stepniak E, Ricci R, Eferl R, Sumara G, Sumara I, Rath M, Hui L, Wagner EF: c-Jun/AP-1 controls liver regeneration by repressing p53/p21 and p38 MAPK activity. Genes Dev 2006, 20: 2306-2314.

9. Passegue E, Wagner EF: JunB suppresses cell proliferation by transcriptional activation of p16(INK4a) expression. EMBO J 2000, 19:2969-2979.

10. Weitzman JB, Fiette L, Matsuo K, Yaniv M: JunD protects cells from p53-dependent senescence and apoptosis. Mol Cell 2000, 6:1109-1119.

11. Jochum W, David JP, Elliott C, Wutz A, Plenk H, Jr, Matsuo K, 
Wagner EF: Increased bone formation and osteosclerosis in mice overexpressing the transcription factor Fra-1. Nat Med 2000, 6:980-984.

12. Sabatakos G, Sims NA, Chen J, Aoki K, Kelz MB, Amling M, Bouali Y, Mukhopadhyay K, Ford K, Nestler EJ, Baron R: Overexpression of DeltaFosB transcription factor(s) increases bone formation and inhibits adipogenesis. Nat Med 2000, 6:985990.

13. Eferl R, Hoebertz A, Schilling AF, Rath M, Karreth F, Kenner L, Amling M, Wagner EF: The Fos-related antigen Fra-1 is an activator of bone matrix formation. EMBO J 2004, 23:2789-2799.

14. McHenry JZ, Leon A, Matthaei KI, Cohen DR: Overexpression of fra-2 in transgenic mice perturbs normal eye development. Oncogene 1998, 17:1131-1140.

15. Karreth F, Hoebertz A, Scheuch H, Eferl R, Wagner EF: The AP1 transcription factor Fra2 is required for efficient cartilage development. Development 2004, 131:5717-5725.

16. Eferl R, Zenz R, Theussl HC, Wagner EF: Simultaneous generation of fra-2 conditional and fra-2 knock-out mice. Genesis 2007, 45:447-451.

17. Kenner L, Hoebertz A, Beil T, Keon N, Karreth F, Eferl R, Scheuch $\mathrm{H}$, Szremska A, Amling M, Schorpp-Kistner M, et al.: Mice lacking JunB are osteopenic due to cell-autonomous osteoblast and osteoclast defects. J Cell Biol 2004, 164:613-623.

18. Karin $\mathrm{M}$ : Inflammation-activated protein kinases as targets for drug development. Proc Am Thorac Soc 2005, 2:386-390; discussion 394-395.

19. Kawai T, Sato S, Ishii KJ, Coban C, Hemmi H, Yamamoto M, Terai $\mathrm{K}$, Matsuda M, Inoue J, Uematsu S, et al.: Interferon-alpha induction through Toll-like receptors involves a direct interaction of IRF7 with MyD88 and TRAF6. Nat Immunol 2004, 5:1061-1068.

20. Rincon M, Derijard B, Chow CW, Davis RJ, Flavell RA: Reprogramming the signalling requirement for AP-1 (activator protein-1) activation during differentiation of precursor CD4 ${ }^{+}$ T-cells into effector Th1 and Th2 cells. Genes Funct 1997, 1: 51-68.

21. Hartenstein B, Teurich S, Hess J, Schenkel J, Schorpp-Kistner M, Angel P: Th2 cell-specific cytokine expression and allergeninduced airway inflammation depend on JunB. EMBO J 2002, 21:6321-6329.

22. Gao M, Labuda T, Xia Y, Gallagher E, Fang D, Liu YC, Karin M: Jun turnover is controlled through JNK-dependent phosphorylation of the E3 ligase Itch. Science 2004, 306:271-275.

23. Meixner A, Karreth F, Kenner L, Wagner EF: JunD regulates lymphocyte proliferation and $\mathrm{T}$ helper cell cytokine expression. EMBO J 2004, 23:1325-1335.

24. Reichardt HM, Kaestner KH, Tuckermann J, Kretz O, Wessely O, Bock R, Gass P, Schmid W, Herrlich P, Angel P, Schutz G: DNA binding of the glucocorticoid receptor is not essential for survival. Cell 1998, 93:531-541.

25. Smolen JS, Hayer S, Schett G, Redlich K, Aringer M, Kollias G, Wagner E, Steiner G: Autoimmunity and rheumatoid arthritis [abstract]. Autoimmun Rev 2004, 3(Suppl 1):S23.

26. Goronzy JJ, Weyand CM: T-cell regulation in rheumatoid arthritis. Curr Opin Rheumatol 2004, 16:212-217.

27. Firestein GS: Evolving concepts of rheumatoid arthritis. Nature 2003, 423:356-361.

28. Kong YY, Feige U, Sarosi I, Bolon B, Tafuri A, Morony S, Capparelli $\mathrm{C}$, Li J, Elliott R, McCabe $\mathrm{S}$, et al.: Activated $\mathrm{T}$ cells regulate bone loss and joint destruction in adjuvant arthritis through osteoprotegerin ligand. Nature 1999, 402:304-309.

29. Lacey DL, Timms E, Tan HL, Kelley MJ, Dunstan CR, Burgess T, Elliott R, Colombero A, Elliott G, Scully S, et al.: Osteoprotegerin ligand is a cytokine that regulates osteoclast differentiation and activation. Cell 1998, 93:165-176.

30. O'Gradaigh D, Compston JE: T-cell involvement in osteoclast biology: implications for rheumatoid bone erosion. Rheumato/ogy (Oxford) 2004, 43:122-130.

31. Yasuda H, Shima N, Nakagawa N, Mochizuki SI, Yano K, Fujise N, Sato Y, Goto M, Yamaguchi K, Kuriyama M, et al.: Identity of osteoclastogenesis inhibitory factor (OCIF) and osteoprotegerin (OPG): a mechanism by which OPG/OCIF inhibits osteoclastogenesis in vitro. Endocrinology 1998, 139:13291337.

32. Wada T, Nakashima T, Hiroshi N, Penninger JM: RANKL-RANK signaling in osteoclastogenesis and bone disease. Trends $\mathrm{Mol}$ Med 2006, 12:17-25.
33. Matsuo K, Galson DL, Zhao C, Peng L, Laplace C, Wang KZ, Bachler MA, Amano H, Aburatani H, Ishikawa H, Wagner EF: Nuclear factor of activated T-cells (NFAT) rescues osteoclastogenesis in precursors lacking c-Fos. J Biol Chem 2004, 279: 26475-26480.

34. Takayanagi H, Kim S, Matsuo K, Suzuki H, Suzuki T, Sato K, Yokochi T, Oda H, Nakamura K, Ida N, et al.: RANKL maintains bone homeostasis through c-Fos-dependent induction of interferon-beta. Nature 2002, 416:744-749.

35. Burrage PS, Mix KS, Brinckerhoff CE: Matrix metalloproteinases: role in arthritis. Front Biosci 2006, 11:529-543.

36. Han Z, Boyle DL, Chang L, Bennett B, Karin M, Yang L, Manning AM, Firestein GS: c-Jun N-terminal kinase is required for metalloproteinase expression and joint destruction in inflammatory arthritis. J Clin Invest 2001, 108:73-81.

37. Smolen JS, Aletaha D, Koeller M, Weisman MH, Emery P: New therapies for treatment of rheumatoid arthritis. Lancet 2007, 370:1861-1874

38. Zenz R, Scheuch H, Martin P, Frank C, Eferl R, Kenner L, Sibilia $\mathrm{M}$, Wagner EF: c-Jun regulates eyelid closure and skin tumor development through EGFR signaling. Dev Cell 2003, 4:879889.

39. Lowes MA, Bowcock AM, Krueger JG: Pathogenesis and therapy of psoriasis. Nature 2007, 445:866-873.

40. Mease P: Psoriatic arthritis update. Bull NYU Hosp Jt Dis 2006 , 64:25-31.

41. Nickoloff BJ: Keratinocytes regain momentum as instigators of cutaneous inflammation. Trends Mol Med 2006, 12:102-106.

42. Nickoloff BJ, Nestle FO: Recent insights into the immunopathogenesis of psoriasis provide new therapeutic opportunities. J Clin Invest 2004, 113:1664-1675.

43. Zenz R, Eferl R, Kenner L, Florin L, Hummerich L, Mehic D, Scheuch H, Angel P, Tschachler E, Wagner EF: Psoriasis-like skin disease and arthritis caused by inducible epidermal deletion of Jun proteins. Nature 2005, 437:369-375.

44. Johansen C, Kragballe K, Rasmussen M, Dam TN, Iversen L: Activator protein 1 DNA binding activity is decreased in lesional psoriatic skin compared with nonlesional psoriatic skin. $\mathrm{Br} J$ Dermatol 2004, 151:600-607.

45. Haider AS, Duculan J, Whynot JA, Krueger JG: Increased JunB mRNA and protein expression in psoriasis vulgaris lesions. J Invest Dermatol 2006, 126:912-914.

46. Hobbs JA, May R, Tanousis K, McNeill E, Mathies M, Gebhardt C, Henderson R, Robinson MJ, Hogg N: Myeloid cell function in MRP-14 (S100A9) null mice. Mol Cell Biol 2003, 23:25642576.

47. Conrad C, Nestle FO: Animal models of psoriasis and psoriatic arthritis: an update. Curr Rheumatol Rep 2006, 8:342-347.

48. Gudjonsson JE, Johnston A, Dyson M, Valdimarsson H, Elder JT: Mouse models of psoriasis. J Invest Dermatol 2007, 127:12921308

49. Keffer J, Probert L, Cazlaris H, Georgopoulos S, Kaslaris E, Kioussis D, Kollias G: Transgenic mice expressing human tumour necrosis factor: a predictive genetic model of arthritis. EMBO J 1991, 10:4025-4031.

50. Jo SJ, Park JY, Yoon HS, Youn Jl: Case of acrodermatitis continua accompanied by psoriatic arthritis. J Dermatol 2006, 33: 787-791.

51. Serarslan G, Guler H, Karazincir S: The relationship between nail- and distal phalangeal bone involvement severity in patients with psoriasis. Clin Rheumato/ 2007, 26:1245-1247. 